\title{
El cambiante mundo de las organizaciones. Hacia una organización saludable
}

\author{
Miguel Ángel Gimeno Navarro, Antonio Grandio, Ana I. Marqués \\ Universitat Jaume I \\ ngimeno@uji.es; agrandio@emp.uji.es; imarques@uji.es
}

Resumen: Nuestro modelo actual de sociedad se balla inmerso en un profundo cambio (re-evolución), que nos está conduciendo hacia un mundo «enredado». Las organizaciones, como subsistemas fundamentales de esa sociedad, tienen el reto de digerir el complejo conjunto de transformaciones que estamos viviendo.

Uno de los patrones que están emergiendo en las organizaciones, como respuesta a este proceso de cambio, es el de la «salud en la organización». El desarrollo de este patrón se concreta en el constructo «organizaciones saludables», que se caracterizan por promover y potenciar la sinergia positiva entre su propio crecimiento, el de sus miembros y el de la comunidad. La construcción de este tipo de organizaciones se ha convertido en uno de los desafíos del mundo organizacional y, por ende, de la propia sociedad. Su viabilidad y concreción pueden determinar, en buena medida, el modelo social del futuro a medio y largo plazo.

El estado de la cuestión es aún demasiado disperso. Se requiere formular un marco referencial que permita orientar el desarrollo de este tipo de organizaciones; un marco que, a su vez, nos ofrezca una conceptualización adecuada para, posteriormente, plantear el desarrollo progresivo de las configuraciones organizativas que posibiliten la evolución integra y bolistica de la salud de la organización, de sus miembros y de la comunidad. Partiendo de la revisión sistemática de información, el presente trabajo ofrece una primera aproximación configuracional en este camino.

Palabras clave: Organización saludable, salud en la organización, calidad de vida laboral, desarrollo organizacional, constructivismo social. 
Abstract: Our present model of society is engaged in a profound change (re-evolution) that is directing us towards a «networked world». Organizations, as fundamental subsystems of this society, have to cope with the challenge of managing the complex transformation frameset we are experiencing.

One of the patterns that is emerging in response to this changing process is health in organization. The development of this pattern draws closer to the construct of healthy organizations, which promote and empower the positive synergy between their own growth, that of their members and that of the community. Building this type of organization has become one of the challenges of the organizational world and, therefore, of society itself. A great deal of the future social model in the mid and long term may depend on their feasibility and concreteness.

The state of the art is still too fragmented. A reference framework needs to be formulated that enables these kinds of organization to be developed. The framework should also conceptualize the organization and then progressively develop organizational configurations that lead to the comprehensive and holistic evolution of the health of the organization, its members and the community. On the basis of a systematic review of information, the present paper uses a configurational approach to make an initial proposal.

Keywords: Healthy organizations, Health in Organizations, Quality in Work Life, Organizational Development, Social Constructivism. 


\section{Planteamiento del problemaः la emergencia del patrón «salud en la organización»}

El marco teórico del constructivismo social sostiene que las organizaciones nos construyen y, al mismo tiempo, nosotros las vamos construyendo, dentro de un proceso interactivo y retroalimentado. En este sentido, Maslow $(1971,2005)$ argüía que cada época ha tenido su propio sistema institucional.

Los diferentes modelos conceptuales de la realidad organizativa han ido surgiendo en sintonía con la evolución histórico-social del momento (Gimeno, 2011). Así, hemos ido interpretando las organizaciones de muy diferentes formas (véanse las metáforas planteadas por Morgan -1986-, o la clasificación de Scott - 2003_, entre otras).

El mundo actual está abierto a una profunda metamorfosis (Ovejero, 2003; Scott, 2003), que entraña el paso de la era industrial a la digital (Tapscott, 2011). La nueva realidad emergente viene marcada por la complejidad, el cambio de valores, la multiculturalidad, y la incertidumbre (Barriga et alii, 2004). Nuestra sociedad se está reorganizando y nuestro mundo cotidiano con ella (Ovejero, 2003). Las ideas dominantes en nuestra civilización desde la Revolución Industrial comienzan a resquebrajarse. Se alteran muchas de las realidades que antes se consideraban certeras. El hecho de que se den, al unísono, muchos y variados desafíos ha llevado a afirmar que nos encontramos en el «límite del caos». Estamos ante una nueva era, la era del mundo «enredado» (networked world), caracterizada por su complejidad. En ella los planes y las estrategias suelen fallar; ahora son más pertinentes y útiles la inmersión, la interacción y la colaboración, la atención y la emergencia, para gestionar tanto las empresas como sus grupos humanos (Grandío, 2011).

Estos cambios que estamos experimentando en el conjunto de nuestra sociedad conllevan también la modificación sustancial del ámbito de las organizaciones laborales (Cascio, 1995; Fuertes, 2002). De hecho, estas se encuentran inmersas en una profunda transmutación, mayor que cualquier otra de las producidas en los últimos 300 años (Kravetz, 1990). Los esquemas organizacionales, sus límites, características, estructuras y procesos han sido cuestionados (Senge, 1990), y son múltiples los retos a los que responder. Scott (2003) considera cinco cambios trascendentales: 1) en la naturaleza de los límites organizacionales, 2) en las estrategias organizacionales, 3 ) en las formas organizacionales, 4) en los componentes organizacionales y, más fundamentalmente, 5) en la manera en que concebimos a las organizaciones. 
Esta nueva realidad de las organizaciones nos obliga a realizar una lectura divergente de estas, más cercana al paradigma de la complejidad y a la teoría general de sistemas (Adkins, 2000; Rodríguez, 1992). Entramos en un periodo de emergencia de patrones de relación desconocidos hasta ahora, en todos los niveles organizacionales, que evidencia la ruptura del modelo organizativo tradicional y el surgimiento de uno nuevo (Gimeno, 2011; Grandío, 2011).

\section{Estrategia vs. atención sostenida}

En el nuevo paradigma de la complejidad, una de las dimensiones principales es la emergencia de nuevas propiedades y fenómenos, los cuales no pueden ser anticipados ni previstos. Esto convierte en caduca la orientación «estratégica» vigente en la que se formulan objetivos y se implementan después a través de pautas más o menos planificadas racionalmente. Cada vez más, en vez de perseguir ciertos objetivos a través de ciertas estrategias premeditadas, las organizaciones tienen que descubrir los patrones emergentes y tratar de entender su sentido mediante la «atención sostenida» sobre el mundo.

Por otro lado, las organizaciones ya no son algo independiente de su entorno, ni de los seres humanos que las conforman. La introducción de la complejidad y de los sistemas autoorganizados adaptativos está reviviendo las ideas expuestas por Morin (1994): en la medida en que las organizaciones deseen sobrevivir y crecer lo han de hacer con y desde su entorno y sus miembros. En este nuevo modelo organizacional los trabajadores pasan de ser considerados un Coste a ser un Recurso (etapa presente), hasta ser vistos como un Componente (ser parte de) (Grandío, 1996); se enfatizan las interacciones entre la organización, su ambiente externo y los trabajadores; se destacan los aspectos técnicos del diseño organizacional relacionados con las necesidades e intereses de los trabajadores, y el uso de modelos de dirección que enfatizan el apoyo y la participación.

Sin embargo, aunque este emerger es cada vez más palpable, continúan predominando los contextos laborales dominados por la gestión «tóxica». En este tipo de contextos el trabajo se experimenta como una obligación, un aburrimiento, o algo alienante (Bestratén y Pujol, 2003; Kets de Vries, 2001; Mejías, et alii 2011; Vilaseca, 2010). Por otro lado, la actual coyuntura socioeconómica está socavando el estado del bienestar y promoviendo, dentro del ámbito laboral, el retorno a esas prácticas sociolaborales tóxicas que ya parecían superadas.

Nos encontramos, así, ante dos tendencias abiertamente opuestas. Por un lado, aquella que, alentada por la situación de crisis y por visiones conservadoras del mundo sociolaboral, defiende modelos tradicionales de gestión (por ejemplo, el taylorismo o el fordismo), en los que se asimila al ser humano al hombre $\mathrm{X}$ 
de McGregor. Por otro, aquella que, dentro del movimiento de re-evolución social expuesto anteriormente, intenta dar respuestas a la propia evolución del ser humano y de la sociedad, y que está impregnada por la visión del hombre Y de McGregor.

Entendemos que esta confrontación es transitoria y natural, siendo fruto de las propias dinámicas de evolución social y laboral. Los cambios de paradigma suponen la exacerbación de las posturas confrontadas, donde el viejo paradigma realiza un último intento por hacer valer los pilares que lo sostienen, mientras el nuevo necesita de ese contraste para alcanzar su propia definición. De acuerdo con diferentes autores (Stiglitz, 2002) la construcción de la «buena sociedad», donde las organizaciones son la base, exige una rehumanización de estas, que debe tener como base moral el bienestar de su gente trabajadora. Por consiguiente, la mejora en las condiciones de bienestar resulta esencial para fomentar la dinámica de cambio organizacional que estamos viviendo. Es por ello que el patrón emergente de la «salud en la organización» se convierte en una de las piedras angulares de la re-evolución organizacional en el nuevo contexto sociocultural y económico que estamos configurando, sustentado en claves culturales diferentes a las del pasado (Gimeno, 2011).

Las empresas van tomando conciencia, progresivamente, de que una parte de su responsabilidad es promover la salud a través de mejores lugares de trabajo (Peiró y Rodríguez, 2008). El cambio en los valores sociales nos muestra que ya no importa tanto la distribución de recursos como el modo de vivir, la forma en que se debe tratar la naturaleza, el medio ambiente y los recursos de que disponemos, entre otros aspectos. La gestión tóxica de las organizaciones, aceptada como inevitable hace unas décadas, ahora produce un aumento en el malestar y un descreimiento generalizado, como muestra el estudio realizado por Towers Watson (Olabarrieta, 2010). Siguiendo las reflexiones de Maslow (1971, 2005), e incluso aceptando la profunda crisis global que estamos experimentando, entendemos que esta nueva época apunta hacia el sistema de las organizaciones saludables y de los seres humanos autorrealizados. El desarrollo de este sistema implica la construcción de entornos laborales que hagan posible y potencien la Calidad de Vida Laboral (ver Figura 1), de forma que las personas evolucionen hacia la realización personal y de la comunidad. Para ello, el ambiente laboral ha de crear las condiciones para que los seres humanos desarrollen lo mejor de sí mismos (González et alii, 1996) y crezcan en su potencial de salud. 
Figura 1. Influencia de algunos elementos de cambio sobre la mejora de la calidad de vida en las empresas

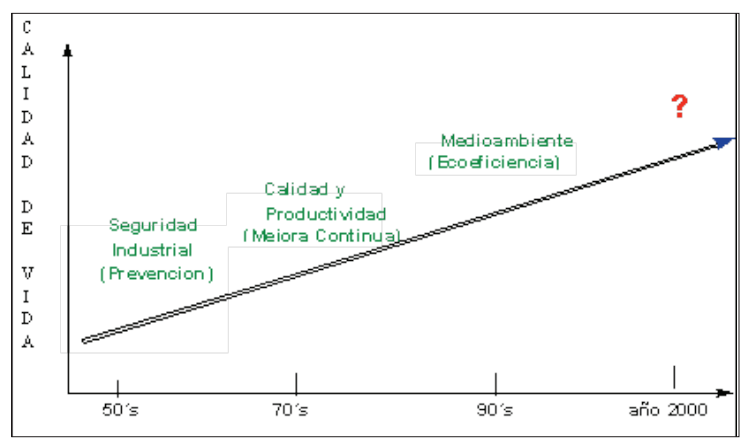

Fuente: González, Yoshimatsu y Cantú, (2005)

La emergencia del patrón de la «salud en la organización» es fruto de la evolución y complejización crecientes de los conceptos de salud y organización. Este patrón hubiera sido impensable desde visiones más cerradas y exclusivas, tanto de la salud como de la organización. Por ello, la creación y desarrollo de la organización saludable se considera como un proceso dinámico e inclusivo, marcadamente evolutivo. A continuación, pasamos a analizar el desarrollo y el estado actual de estos dos conceptos básicos. Posteriormente se analiza la sinergia que entre ambos se produce y que se concreta en el constructo de la organización saludable.

\section{Fundamentación teórica: de la protección a la promoción de la salud laboral}

\subsection{La evolución del concepto de salud}

La salud laboral recibe cada vez más atención por parte de los investigadores, en buena medida por los importantes costes que la falta de salud en el trabajo puede tener tanto para las organizaciones como para las personas que las conforman (Caulfield, 2006; Chappell y Di Martino, 2006; European Agency for Safety and Health at Work-OSHA-, 2010; O'Leary-Kelly et alii, 1996; Organización Internacional del Trabajo et alii, 2002; Organización Internacional del Trabajo OIT-, 2005; Paoli y Merllié, 2001; Parent-Thirion et alii, 2007; Weiler, 2006; Wilson et alii, 2004; Zapf et alii, 1996, entre otros). El deterioro en la salud de las personas puede acarrear un aumento en la tasa de absentismo, un decremento en los niveles de rendimiento y una pérdida en la calidad de vida de las personas y de 
su entorno. Por el contrario, la promoción de la salud en el contexto laboral suele conllevar mejoras en el rendimiento, así como conductas voluntarias favorables para la organización (Buck Consultants, 2009; Danna y Griffin, 1999; Peiró y Rodríguez, 2008; Romay et alii, 2003). Si bien estas correlaciones lineales están empezando a ser cuestionadas (MacIntosh et alii, 2007) y constituyen una de las razones del aumento en la complejidad del mundo organizacional.

La Organización Mundial de la Salud (OMS) define la salud como un estado completo de bienestar físico, psicológico y social (OMS, 1978). Esta definición constituye el fin del reduccionismo que había supuesto la consideración de la salud como la ausencia de enfermedad. El triunfo del modelo biopsicosocial incorpora factores físicos, psicológicos y sociales, no solo evitando la enfermedad sino también fomentando la adaptación y el crecimiento de varios roles y entornos (Tetrick, 2002), asentándose en la Teoría General de Sistemas (Traid et alii, 2005), y constituyendo un acercamiento más holístico a la salud.

A finales de los años sesenta se estaban produciendo importantes cambios en la consideración de la salud y en su promoción. La aproximación higienista, que primó durante la primera mitad del siglo xx, se centró en esterilizar y desinfectar. Esta forma de entender la salud chocaba frontalmente con las nuevas propuestas en la materia. Se hizo imprescindible la elaboración de herramientas más complejas y holísticas. Incorporar esta complejidad y la democracia - mediante la participación - a la práctica de la salud pública son retos que se hacen cada vez más evidentes durante los años 80 y que conducen a hacia la «nueva salud pública» (Álvarez-Dardet, 2008).

Desde el modelo biopsicosocial la salud pasa a ser contemplada desde el prisma pluridimensional de la calidad de vida y del bienestar individual y social, y sigue un esquema integral de las tres vertientes en que la vida se presenta (García et alii, 2000): lo biológico/orgánico, lo psicológico/afectivo y lo conductual/de interacción social. Esta aproximación ofrece una visión del ser humano en su globalidad y en relación con su entorno. Aporta una idea de salud entendida desde la normalidad utópica (Lluch, 1999). Muestra una tendencia hacia una situación deseable u óptima. Además, introduce la subjetividad de la persona en la valoración de su salud completa (Lluch, 1999).

El concepto de salud se amplifica, integrando sociedad y persona, de forma que se puede considerar como aquel conjunto de condiciones físicas, psíquicas y sociales que permiten que la persona desarrolle y ejerza todas sus facultades en armonía con su propio entorno. Esto supone que, además de cambios a nivel individual, resulten necesarios cambios sociales, organizacionales y políticos (León, 2000). La salud de las personas, la de las comunidades y la del entorno natural 
pasan a ser consideradas como interdependientes. Esta idea es la que ha llevado a desarrollar el Movimiento de las Ciudades Saludables y a que se consolide el movimiento de la New Public Health (OMS, 1986). En la Conferencia de la OMS en Ottawa en 1986, la salud se define como "los procesos que favorecen el control de los individuos y grupos sobre las variables que condicionan su propia salud", y «para conseguir un estado de bienestar físico, mental y social completos, una persona o un grupo han de poder identificar y lograr sus aspiraciones, satisfacer sus necesidades y cambiar con el medio ambiente o adaptarse a él».

Ahora se presenta al ser humano como un todo y a su salud como un resultado de aspectos biológicos, psicológicos y sociales que interactúan en los diversos escenarios en los que se desarrolla la vida cotidiana de las personas (León, 2000).

En el esfuerzo por promocionar la salud y el bienestar para todas las personas se articula el constructo de la «calidad de vida», el cual permite una perspectiva más operativa y, a priori, más medible (Lluch, 1999). La calidad de vida, identificada con indicadores objetivos como los ingresos, la salud, las condiciones ambientales, el empleo, la vivienda, etc, ha sido mimetizada con el bienestar social, ligándola con indicadores socioeconómicos macrosociales (García et alii, 2000).

El cambio producido en el campo de la salud con el surgimiento y consolidación del modelo biopsicosocial y de su promoción desde el constructo de calidad de vida laboral ha repercutido en la perspectiva organizativa, tal y como veremos en el apartado siguiente. Así, se observan dos procesos de desarrollo y cambio: uno, parcial, relacionado con la actividad preventiva en las empresas, y otro, más amplio, vinculado a la propia trasmutación del mundo organizacional.

En relación con el desarrollo preventivo, la forma de actuar frente a los riesgos laborales en el ámbito de las organizaciones ha ido experimentando una evolución a lo largo de todo el siglo xx. Esta evolución ha sido inclusiva; cada una de las etapas ha ido ampliando el área de actuación preventiva, dando respuesta a nuevas realidades del mundo laboral y de la salud. En la actualidad, se están empezando a considerar las condiciones de trabajo como el objeto de la actividad preventiva (Ley de Prevención de Riesgos Laborales, 1995, 2003). Este desarrollo se centra en lo que la OMS (2010) conoce como actuación para la protección de la salud.

Por su parte, la aproximación centrada en la transmutación organizacional guarda relación con la promoción de la salud en el ámbito laboral. Dicha promoción supone capacitar a las personas para el control sobre su salud y sus determinantes, y por lo tanto para mejorar su salud (Stresa Declaration on Workers Health, 2006; OMS, 1997, 2010). Es desde este aproximación que el patrón «salud en la organización» cobra pleno sentido. 


\subsection{La salud desde el ámbito organizativo}

El desarrollo máximo de la salud supone que tanto la sociedad como sus subsistemas (organizaciones, instituciones, etc.) presenten unas estructuras y sistemas de valores que la fomenten (León, 2000). Esto significa que el ser humano solo puede alcanzar la máxima salud que el contexto social y laboral en el que vive le posibilita (León y Jarana, 1992). Así pues, las condiciones sociales y organizacionales juegan un papel básico en el potencial de salud y desarrollo de las personas que en ellas viven.

Ahora bien, las organizaciones, en general, se han orientado principalmente hacia el logro de sus metas y la obtención de aquellos beneficios para los que han sido concebidas. Esto las ha alejado, durante mucho tiempo, de la atención por la salud de sus miembros y de la comunidad en la que viven, al entender que este no era uno de sus cometidos. Fue en la década de 1940 cuando se observó que la mejora de las condiciones laborales, la promoción de la seguridad y la salud, el fomento de la participación y la atención a las relaciones sociales, entre otros aspectos, resultaban beneficiosos para la organización, en tanto que reducían conductas disruptivas por parte de sus miembros y promovían conductas orientadas hacia las metas organizacionales.

Entre las décadas de 1960 y 1980 se despliega el movimiento de la calidad de vida laboral, que dota de relevancia a los entornos de trabajo saludables. En sus orígenes, enfatizó la necesidad de encontrar formas de organización y estilos de dirección que garantizaran las condiciones para que, en el trabajo, los seres humanos desarrollasen lo mejor de sí mismos (Mateu, 1984; Navajas, 2003). Sin embargo, con el tiempo fue tomando preponderancia la perspectiva centrada en la calidad de vida del entorno de trabajo (Blanco y Chacón, 1985; Parra, 1993; Segurado y Agulló, 2002). Esta derivación supuso la pérdida de la esencia del movimiento y su disolución (Navajas, 2003).

En las últimas dos décadas, el énfasis en la calidad del ambiente laboral como elemento esencial para la salud organizacional (Cox, 1992) y la relevancia de los factores organizacionales en las relaciones salud-trabajo (Vandenberg et alii, 2002) han contribuido al resurgir del constructo «calidad de vida laboral», ubicándolo como elemento central en el actual proceso de metamorfosis organizacional (González et alii, 1996). El denominado Movimiento de la Organización de Trabajo Saludable sostiene dos asunciones (Bond, 2004; Schmidt et alii, 2000): 1) es posible identificar un conjunto de factores del puesto y de la organización que la caracterizan como saludable; 2) la creación de este tipo de lugar de trabajo debería favorecer mayor salud, seguridad, y productividad en los trabajadores, lo que debería saldarse incrementando los beneficios y la competitividad en el mercado. 
En este tercer periodo se aprecian dos velocidades en la aproximación de las organizaciones a la promoción de la salud (ver tabla 1). La vía que surgió en primer lugar es más pragmática. La forma de plantear el desarrollo de entornos de trabajo saludables es más reactiva («acciones ante») que proactiva («promoción de»). Presenta una visión limitada de la salud, más centrada en su protección, y no contempla el desarrollo integral del trabajador. La salud es importante como herramienta de gestión de recursos humanos, no es un fin en sí misma; su finalidad continúa siendo la productividad y la eficacia organizacional, que, se entiende, puede lograrse mejor disponiendo de trabajadores sanos en entornos seguros (Salanova, 2008). La organización no se abre a sus miembros, sino que introduce ciertas prácticas que entiende que le pueden ser útiles. Además, la fuente del problema suele situarse en el individuo antes que en la organización. El concepto, desde esta perspectiva, sería el de «salud organizativa». Esta vía suscita resquemores entre diferentes autores, que sospechan que no significa un cambio real, sino más de lo mismo (Aguirre y Guerrero, 2005; Banerjee, 2007; Patrus, 2007; Píriz, 2009; Senge, 2001, entre otros).

Tabla 1. Diferencias entre la perspectiva de la salud organizativa y la perspectiva de la salud en la organización. Elaboración propia

\begin{tabular}{|c|c|}
\hline Perspectiva salud organizativa & Perspectiva salud en la organización \\
\hline $\begin{array}{l}\text { + Concepto: salud organizativa } \\
+ \text { Acción: reactiva (protección ante) } \\
+ \text { Actuaciones orientadas a prevención y } \\
\text { protección frente a riesgos } \\
+ \text { Acciones esporádicas } \\
+ \text { Aproximación: fraccionada } \\
+ \text { Lógica: crecimiento } \\
+\quad \text { Visión simple del concepto de salud (coste } \\
\text { o a lo sumo inversión) } \\
+ \text { Objetivos: diferencia entre final (efectivi- } \\
\text { dad organizativa -productividad y compe- } \\
\text { titividad-) e instrumental (salud empleado) } \\
+\quad \text { Primero la organización, luego el empleado } \\
+ \text { Empleado: consideración instrumental } \\
\text { + Foco: empleados con problemas }\end{array}$ & $\begin{array}{l}\text { + Concepto: salud en la organización } \\
+ \text { Acción: proactiva (promoción de) } \\
+ \text { Actuaciones orientadas al desarrollo de } \\
\text { las potencialidades de sus miembros } \\
+ \text { Acciones integradas } \\
+ \text { Aproximación: sistémica } \\
+ \text { Lógica: desarrollo } \\
+ \text { Visión compleja del concepto de salud } \\
\text { (fin teleológico) } \\
+ \text { Objetivos: coincidentes (desarrollo mu- } \\
\text { tuo organización-persona) } \\
+ \text { Empleado y organización en sintonía } \\
+ \text { Empleado: consideración finalista } \\
+ \text { Foco: todos los empleados }\end{array}$ \\
\hline
\end{tabular}

La segunda vía se está consolidando poco a poco, y presenta una visión más amplia, sistémica y compleja del concepto de salud; también más utópica y, tal vez por ello, más cercana al modelo organizacional, social y humano emergente. Más que la protección, su objetivo prioritario es la promoción de la salud interdepen- 
diente de la organización, los trabajadores, y la comunidad. El fin es el desarrollo mutuo de los tres, desde la sintonización. El concepto, desde esta vía, sería el de «salud en la organización». La necesidad de diferenciar entre salud organizativa y salud en la organización ha sido señalada por MacIntosh, MacLean y Burns (2007).

Por otro lado, tomando referencias de la Declaración de Yakarta, la Declaración de Stresa, el Plan Global Compacto y el Plan Global de Acción para la Salud de los Trabajadores, en la creación de ambientes de trabajo saludables se ha de tener en cuenta el vínculo existente entre el espacio de trabajo y la comunidad.

Estas consideraciones llevan a la OMS (2010) a definir un entorno de trabajo saludable en los siguientes términos:

aquel en el que los trabajadores y el personal superior colaboran en la aplicación de un proceso de mejora continua para proteger y promover la salud, la seguridad y el bienestar de todos los trabajadores y la sostenibilidad del lugar de trabajo, teniendo en cuenta las siguientes consideraciones establecidas sobre la base de las necesidades previamente determinadas: temas de salud y de seguridad en el entorno físico de trabajo; temas de salud, seguridad y bienestar en el entorno psicosocial de trabajo, con inclusión de la organización del trabajo y de la cultura laboral; recursos de salud personal en el lugar de trabajo, y maneras de participar en la comunidad para mejorar la salud de los trabajadores, sus familias y otros miembros de la comunidad (p. 16).

\section{Propuesta de marco de referencia para la construc- ción de organizaciones saludables}

En otros lugares (Grandío, 1996; Grandío y Chiva, 1997; Grandío et alii, 1998) hemos elaborado un marco teórico evolutivo basado, por un lado, en la conocida jerarquía de necesidades de Maslow $(1959,1971)$ y, por otro, en los «mecanismos de coordinación» organizativos de Mintzberg (1984). La idea básica que subyace tras dicho marco es que estos mecanismos son la manifestación «natural» de alguna de las necesidades de Maslow y que, por lo tanto, cabe encontrar un «isomorfismo jerárquico entre cada necesidad y cada mecanismo de coordinación».

Mintzberg distinguió seis mecanismos de coordinación: la adaptación mutua (coordinación del trabajo mediante la simple comunicación informal); la supervisión directa (coordinación al responsabilizarse una persona del trabajo de los demás, dirigiendo y controlando sus acciones); la normalización, que presenta cuatro formas básicas: normalización de operaciones o procesos de trabajo (es- 
pecificar lo más detalladamente posible el contenido de cada trabajo); la normalización de outputs o resultados (especificación de las metas, objetivos o fines que lograr); la normalización de habilidades (especificando los conocimientos, competencias y habilidades necesarios para realizar las tareas), y la normalización de normas (la cultura o ideología que provee de una forma homogénea de abordar las tareas).

En dicho marco se hicieron algunos ajustes y adiciones. En primer lugar, y respecto a la jerarquía de Maslow, se utilizaron dos subdivisiones ya contempladas por él mismo. Por un lado, distinguió en las necesidades de estatus o de ego entre la de logro y la de estatus propiamente dicha. Por otro, encontró dos tipos de autorrealización: la meramente sana (a la que denominó Y) y la trascendente (a la que denominó Z). Por otro lado, respecto a los mecanismos de Mintzberg, y aunque este autor no los contempla, hemos creído conveniente diferenciar dos tipos de normalización de normas: por un lado, la cultura o ideología (ligada a las necesidades sociales y bautizada aquí como «normalización social»); por otro, la misión o servicio (ligada al papel de satisfacción de necesidades de la comunidad social en la que la empresa está inmersa y con la que mantiene relaciones esenciales de interdependencia).

Como hemos insinuado, entendemos que este marco conceptual resulta oportuno en el intento de esbozar el marco referencial de las organizaciones saludables, ya que interpreta que cada configuración organizativa y su correspondiente mecanismo de coordinación son consecuencia de un determinado nivel de necesidades (ver tabla 2).

A pesar del trabajo realizado a lo largo del siglo $\mathrm{xx}$, hoy en día no sabemos bien cómo construir una organización saludable. No hay un conjunto definido y cerrado de recursos o técnicas que sirva por igual para todas las organizaciones (Salanova, 2008), si bien parece aceptado que debería ser posible distinguir entre sistemas de trabajo saludables y no saludables (Cooper y Williams, 1994; Cox, 1988; Lowe et alii, 2003; Murphy y Cooper, 2000; Sparks et alii, 2001). Existen, eso sí, amplios listados de acciones que implementar para crear una organización saludable (Vandenberg et alii, 2002). 
Tabla 2. Configuraciones organizativas, modos de coordinación y necesidades

\begin{tabular}{|c|c|c|c|c|}
\hline $\begin{array}{c}\text { Configuración } \\
\text { organizativa }\end{array}$ & $\begin{array}{l}\text { Modo de } \\
\text { coordinación }\end{array}$ & $\begin{array}{l}\text { Parte fundamental de } \\
\text { la organización }\end{array}$ & $\begin{array}{l}\text { Necesidades } \\
\text { (Maslow) }\end{array}$ & $\begin{array}{c}\text { McGregor y } \\
\text { Maslow }\end{array}$ \\
\hline Emprendedora & Supervisión directa & Ápice estratégico & Fisiológicas & \multirow{5}{*}{$\mathrm{X}$} \\
\hline Maquinal & $\begin{array}{c}\text { Normalización de } \\
\text { operaciones }\end{array}$ & Tecnoestructura & Seguridad & \\
\hline $\begin{array}{l}\text { Socialización/ } \\
\text { Sindicación }\end{array}$ & $\begin{array}{l}\text { Normalización } \\
\text { social (sinapsis } \\
\text { fuerte-cerrada) }\end{array}$ & $\begin{array}{l}\text { Sindicato/grupo } \\
\text { Informal. Defensa de } \\
\text { intereses. Centrípeto }\end{array}$ & Sociales & \\
\hline Diversificada & $\begin{array}{l}\text { Normalización de } \\
\text { resultados }\end{array}$ & $\begin{array}{l}\text { Línea media media } \\
\text { (mandos intermedios) }\end{array}$ & Logro & \\
\hline Profesional & $\begin{array}{l}\text { Normalización de } \\
\text { habilidades }\end{array}$ & $\begin{array}{l}\text { Núcleo de } \\
\text { operaciones } \\
\text { (empleados) }\end{array}$ & Estatus & \\
\hline $\begin{array}{l}\text { Innovadora } \\
\text { (Adhocrática) }\end{array}$ & Adaptación mutua & $\begin{array}{c}\text { Staff de apoyo } \\
\text { (I+D, Asesorías } \\
\text { de diversos tipos, } \\
\text { proyectos) }\end{array}$ & Autorrealización & Y \\
\hline Misionaria/ & $\begin{array}{c}\text { Normalización de } \\
\text { Normas (Sinapsis } \\
\text { débil-abierta) }\end{array}$ & $\begin{array}{l}\text { Ideología-Misión } \\
\text { (Servicio) centrífuga }\end{array}$ & Trascendencia & Z \\
\hline
\end{tabular}

Fuente: elaboración propia a partir de Grandio (1998).

Fruto de esa indefinición y ambigüedad han imperado las propuestas parciales y restrictivas. En su mayoría se han centrado en una visión reactiva de la salud laboral y se han restringido al «contexto inmediato de trabajo», normalmente al puesto de trabajo, sin considerar el sistema de organización. Las actuaciones desarrolladas para promover la seguridad y la salud en el trabajo han sido, esencialmente, de carácter técnico y orientadas a aspectos específicos del trabajo (reducción de los niveles de ruido, obtención de equipos de protección individual, protección de riesgos en las máquinas, etc.). No es que dichas intervenciones no resulten oportunas o convenientes; lo que ocurre es que dichas actuaciones son puntuales y no suelen formar parte de ninguna cultura de promoción de la seguridad y la salud en el contexto laboral.

No ha sido hasta la presente década cuando se han propuesto modelos teóricos (Salanova, 2008; Vandenberg et alii, 2002; Wilson et alii, 2004, entre otros) que intentan dar coherencia a esas relaciones de variables y a esas propuestas parciales. El nivel de desarrollo de estos modelos varía considerablemente. Wilson et alii (2004) destacan tres aspectos de los modelos sobre los que existe bastante consenso: el diseño del puesto, el clima organizacional y el futuro en el puesto. 
El problema que presentan estos modelos en su inmensa mayoría es que se centran en la visión limitada de la salud propia de la salud organizacional; además, carecen de la visión dinámica y evolutiva que se precisa para el desarrollo de organizaciones saludables. Por otra parte, solo consideran el nivel organizativo de forma fraccionada, a través de prácticas aisladas. Entendemos que la organización debería ser considerada de forma holística, como un sistema; por dicho motivo sostenemos que la aproximación más adecuada es la configuracional, que permite aprehender el conjunto de la organización (Mintzberg, 1991). Otra de las limitaciones detectadas en estos modelos es su consideración de la salud como un estado final; sin embargo, el análisis de la literatura nos indica que es más fructífero contemplarla como un continuo (Adkins, et alii, 2000; Lowry y Hanges, 2008).

Tomando como referencia los postulados básicos de la OMS en materia de salud $(1986,1998)$, una estrategia básica para la promoción de la salud es la de facilitar que todas las personas puedan desarrollar su completo potencial como seres humanos. Para ello se han de crear, entre otros aspectos, entornos que faciliten ese crecimiento personal; entornos de apoyo que permitan a las personas llevar a cabo todas sus potencialidades. Así, las organizaciones, en tanto que comunidades, para hacerse saludables deben ir incrementando su control sobre los determinantes de la salud y el bienestar. Cuantas más potencialidades para el desarrollo de la salud —en sentido amplio — ofrezca una organización, mayor nivel de salud en la organización tendrá.

Según este símil con los planteamientos de la OMS $(1986,1998)$, una organización empoderada para su salud y bienestar sería aquella en la que sus miembros y la propia organización aplican conjuntamente sus habilidades y recursos en esfuerzos colectivos orientados a abordar la realización personal y a satisfacer sus necesidades respectivas. Este proceso de empoderamiento permite expresar tanto a las personas como a la propia organización sus necesidades y objetivos, y establecer y llevar a cabo acciones para hacerles frente.

Cabría diferenciar entre el empoderamiento para el bienestar de la persona y el empoderamiento para el bienestar de la organización. El primero haría referencia a la capacidad de la persona para tomar decisiones y controlar su propia vida. El segundo implica la actuación colectiva de los miembros de la organización con el fin de conseguir una mayor influencia y control sobre los determinantes del bienestar, la salud y la calidad de vida en su organización.

La promoción de la salud y el bienestar supone responsabilizar tanto a la persona como a las estructuras sociales y al medio que las rodea y en el que se desarrollan. Además, favorece la actuación para el crecimiento en bienestar y no solo 
para su prevención o paliación. Para la promoción se han de considerar «aquellos recursos disponibles para la vida diaria» (OMS, 1986). El objetivo primordial de toda acción que pretenda promocionar la salud y el bienestar de una persona o de una colectividad será el de que esos medios o recursos existan y sean accesibles, pues de lo contrario las personas no pueden alcanzar su máximo potencial de salud y bienestar.

Considerando los análisis y aportaciones hasta aquí realizados, y fruto de la revisión sistemática de documentación, se plantea un modelo evolutivo de organización saludable, que contempla, en esta primera aproximación, cuatro momentos o estadios. Estos momentos conformarían el marco referencial para la construcción de organizaciones saludables. Cada momento superior incluye al que le precede, pero en él emergen nuevos patrones que lo hacen más completo y complejo. En esa progresión se van asumiendo dinámicas que contribuyen a una salud más completa, tanto para los seres humanos como para las propias organizaciones y las comunidades en las que se integran.

1. Aproximación desde el trabajo decente: este marco responde a la visión del trabajador como un recurso que explotar. Plantea respuestas y soluciones que inciden sobre dos de los niveles de necesidades básicas de los seres humanos, a saber: fisiológicas y de seguridad. Preventivamente, las actuaciones que propone se centran en aspectos de seguridad e higiene, más relacionados con la salubridad que con la salud. La configuración organizativa es emprendedora o maquinal; el modo de coordinación la supervisión directa o la normalización de operaciones. Responde a un modelo organizacional de sistema cerrado racional y correlaciona estrechamente con el paradigma de la planificación estratégica en management (Ansoff, 1965, Chandler 1962).

2. Aproximación desde los recursos y capacidades: desde esta perspectiva se ve al trabajador como un recurso al que «cultivar». Plantea respuestas a las necesidades que se corresponden con los niveles social, de logro y de estatus de baja estima (este último hace referencia al respeto de los demás hacia uno mismo y a la necesidad de logros). Preventivamente, se incluye la perspectiva de ergonomía del puesto y de sistemas. La configuración organizativa es la de socialización, diversificada o profesional; el modo de coordinación se basa en la normalización social, de resultados y de habilidades, según el grado de desarrollo. Responde, dependiendo de ese grado, a un modelo organizacional de sistema racional abierto, o de sistema natural cerrado. Podríamos distinguir en ella dos subestadios: en primer lugar el del paradigma de la dirección estratégica, que sustituyó al de la planificación, mayoritariamente vigente hoy en día en muchos ámbitos; se trata de un enfoque centrado en el entorno exterior a la empresa y ligado a la moti- 
vación de logro, normalización de resultados y organización diversificada, cuyo representante más conspicuo podría considerarse Michael Porter (1987). En segundo lugar, el subestadio del paradigma de recursos y capacidades propiamente dicho (Barney, 1991), más ligado a las necesidades de estatus, la normalización de habilidades y la organización profesional (la meritocracia), con su énfasis en el entorno interno y las competencias internas (core competences).

3. Aproximación desde la responsabilidad social de la empresa: en este tercer estadio también podríamos distinguir dos subestadios: el interno y el externo. En el primero se plantea una visión del trabajador como cliente interno con necesidades que considerar. Las necesidades que se cubren son las de estatus de alta estima (supone la necesidad de respeto por uno mismo, de amor propio) y las de autorrealización. Preventivamente, el nivel inclusivo lleva a considerar también ciertas condiciones de trabajo, relacionadas con niveles bajos de participación, autonomía y responsabilidad; se introduce la psicosociología, pero desde la perspectiva negativa. La configuración organizativa que predomina es la profesional y la innovadora; el modo de coordinación fluctúa entre la normalización de habilidades y la adaptación mutua. Puede responder tanto a un modelo organizacional de sistema racional abierto como a uno de sistema natural cerrado.

En el segundo subestadio (el externo), se plantea una visión del trabajador como miembro de una comunidad, pero se contempla explícitamente a la comunidad externa como el ecosistema en la que se encuentra la organización. La empresa tiene una responsabilidad para con la sociedad donde está inscrita, aunque persigue sus propias metas «respetando» las comunidades internas y externas. Las necesidades que se cubren, al igual que en el caso anterior, son las de estatus de alta estima y autorrealización. Preventivamente, se tiene una perspectiva similar a la del subestadio anterior; lo mismo puede decirse de la configuración organizativa que predomina, del modo de coordinación y del modelo organizacional.

4. Aproximación integral desde la misión de la empresa: desde esta aproximación se presenta al empleado como un ser humano con el que desarrollarse mutuamente. En este sentido, la necesidad experimentada a la que se presta atención es la de trascendencia. La empresa existe porque es necesaria, y esta necesidad social, económica y humana es la justificación de su existencia: es su misión. La prevención es proactiva y considera no solo los riesgos sino también los factores de desarrollo del trabajador; la psicosociología se aplica en su sentido positivo. La configuración organizacional que predomina es la misionaria, y el modo de coordinación la normalización de normas, entendida esta como una ideología centrífuga orientada al servicio integral a la comunidad (interna y externa) y a la sociedad. 
Entendemos, desde este modelo evolutivo, que las organizaciones saludables son aquellas que manejan de forma activa la promoción continua del bienestar físico, mental y social de los seres humanos que las conforman, y de la comunidad en la cual se encuentran inmersas, en armonía con su propio beneficio, integrando la sostenibilidad propia y la de su entorno. Pero, en este último estadio, son aún más que esto: hacen emerger la autorrealización dentro y fuera de ellas, constituyéndose en verdaderas catalizadoras del cambio y desarrollo sostenibles hacia potencialidades humanas aún no definidas con precisión. Un paradigma próximo a este estadio podría verse en la organización que aprende de Peter Senge (1990) y sus «cinco disciplinas», una aproximación a la empresa desde la dinámica de sistemas, que podría considerarse una evolución natural de la teoría de sistemas (Bertalanffy, 1940). En este sentido, a modo tentativo, y para investigaciones futuras, consideraríamos deseable contemplar un estadio adicional con su mecanismo de coordinación correspondiente (no contemplado por Mintzberg), basado en la idea de alineamiento que Peter Senge incluye en dicha obra. Es un concepto próximo al de flujo (flow) de Mihalyi Csikszentmihalyi (1996). Probablemente sea una dimensión muy relevante hacia la que orientar nuestras investigaciones futuras.

Para finalizar, cabe señalar que las posibilidades o prácticas que deciden activar las diferentes organizaciones son las que determinan su configuración organizativa. Según sea esta última, se sintonizará o no con una determinada necesidad del ser humano, pudiendo establecerse un continuo de crecimiento, desde la organización tóxica hacia la organización saludable. Así, las organizaciones son el reflejo operativo de la forma en que sus miembros entienden la salud, y siguen una «ruta evolutiva» que arranca en las concepciones más simples (y probablemente cercanas al ámbito tóxico) hasta aquellas más complejas y realizadoras para el ser humano. Es desde esta perspectiva de evolución dinámica inclusiva desde la que consideramos que se ha de concebir y plantear el modelo de construcción de organizaciones saludables. Es también el marco en el que planeamos desarrollar a corto y medio plazo instrumentos empíricos de medida que puedan auxiliarnos en la mejora de este mundo emergente de las organizaciones.

\section{Bibliografía}

Adrins, J. A.; Quick, J. C., y Moe, K. O. (2000). «Building world-class performance in changing times». En Murphy, L. R. y Cooper, C. L. (eds*) (2000). Healthy and productive work. An international perspective. Londres: Taylor \& Francis. 
Aguirre, L. R. y Guerrero, J. L. (2005). Calidad como estilo de vida. En línea: <http://www.gestiopolis.com/canales5/emp/pymecommx/46.htm>.

Álvarez-Dardet, C. (2008). Promoción de la salud. En línea: <http://perso. wanadoo.es/aniorte_nic/ apunt_polit_plan_5.htm >.

Ansoff, I. (1965). Corporate Strategy. Mc Graw-Hill, New York.

BARNEY, J.B. (1991). «Firm resources and sustained competitive advantage». Joumal of Management. (17).

Banerjee, S.B. (2007). Corporate Social Responsibility: The Good, the Bad, and the Ugly. Northampton, MA: Edward Elgar.

Barriga, S.; Trujillo, I*; Medina, S. y León, J.M. (2004). «Globalización y calidad de vida en la sociedad del bienestar: los servicios sociosanitarios». Encuentros en Psicología Social, 2(1):125-129.

Bertalanffy, V. (1940). Vom Molekül zur Organismenwelt, Potsdam: Akademische Verlagsgesellschaft Athenaion.

Bestratén, M. y Pujol, L. (2003). NTP 643: Responsabilidad social de las empresas $(I)$ : conceptos generales. Instituto Nacional de Seguridad e Higiene en el Trabajo. En línea: <http://www.mtas.es/insht/ntp/ntp_643.htm>.

Blanco, A. y Chacón, F. (1985). «La evaluación de la calidad de vida». En Morales, Blanco, Huici y Fernández, Psicología social aplicada. Bilbao: Desclée de Brouwer.

Bond, F.W. (2004). «Getting the balance right: the need for a comprehensive approach to occupational health». Work E Stress, 18(2):146-148.

Buck Consultants (2009). Working well: A global survey of health promotion and workplace wellness strategies: Survey report. En línea: <https://www.bucksurveys.com/bucksurveys/product/tabid/139/p-51-working-well-a-globalsurvey-of-health-promotionand-workplace-wellness-strategies.aspx $>$.

Chandler, A. (1962). Strategy and Structure: Chapters in the History of the American Industrial Enterprise. Cambridge, MA: MIT Press

Cascio, W.F. (1995). «Whither Industrial and Organizational Psychology in a Changing World of Work?». American Psychologist. 50(11). APA.

Caulfield, N. (2006). Violencia en el trabajo en América del Norte. Washington: Secretariado de la Comisión para la Cooperación Laboral.

Chappell, D. y Di Martino, V. (2006). Violence at work, Third edition. Ginebra: OIT.

Cooper, C.L. y Williams, S. (1994). Creating bealthy work organizations. Chichester, UK: Wiley.

Cox, T. (1988). «Editorial: Organizational health». Work E Stress, 2:1-2. 
- (1992). «Occupational health: the control of psychosocial and organizacional hazards at work», Congreso Safety \& well-being at work: A human factors approach. Lougborough.

Csikszentminalyi, M. (1996). Creativity: Flow and the Psychology of Discovery and Invention. New York: Harper Perennial.

Danna, K. y Griffin, R. W. (1999). «Health and well-being in the workplace: a review and synthesis of the literature». Journal of Management. 25(3):357384.

European Agency for Safety and Health at Work (OSHA). (2010). Workplace Violence and Harassment: a european picture. En línea: <http://osha.europa.eu/en/publications/reports/violence-harassmentTERO09010ENC $>$. [Consultado el 10 de febrero de 2011]

Fuertes, F. (2002). Cambio y desarrolloorganizacional. Barcelona: Universitat Oberta de Catalunya.

García, A. M.; Benavides, F., y Ruiz-Frutos, C. (2000). «Salud Laboral». En F. G. Benavides, C.; Ruiz-Frutos, y García, A. M. (eds.). Salud laboral: conceptos y técnicas para la prevención de riesgos laborales, 49-50. Barcelona: Masson.

Gimeno, M. A. (2011). Organizaciones saludables: su relación con el bienestar psicológico. Tesis Doctoral, en preparación. Universitat Jaume I.

González, M. A.; Yoshimatsu, T., y Cantú, D. F. (2005). Ensayo sobre cómo mejorar la calidad de vida en las empresas. En línea: <http://www.sht.com.ar/ archivo/Management/calidad.htm $>$.

González, P.; Peiró, J. M. y Bravo, M. J. (1996). «Calidad de vida laboral». En J. M. Peiró y F. Prieto (eds.). Tratado de psicología del trabajo. Vol.2, Aspectos psicosociales del trabajo. Madrid: Síntesis.

Grandío, A. (1996). Empresa, mercado y necesidades: una sintesis en ciencias sociales. Tesisdoctoral. Universitat Jaume I, Castelló.

- (2011). «La sociedad en red y los recursos humanos (I)». En Agut, S.; GranDío, A., y Peris, R. (eds.). Los recursos bumanos en el ámbito turístico. Claves para su gestión en un mundo en red. Madrid: Síntesis.

Grandío, A. y Chiva, R. (1997). Organization as Learning: a Comprehensive Evolutionary Framework. VII Congreso Hispano-Francés de la Asociación Europea de Dirección de Empresas (AEDEM). Lleida.

Grandío, A.; Chiva, R., y Montesinos, C. (1998). Aprendizaje organizativo: construcción de un instrumento de investigación. VIII Congreso HispanoFrancés de la Asociación Europea de Dirección de Empresas (AEDEM). Las Palmas de Gran Canaria. 
Kets de Vries, M.F.R. (2001). "Creating authentizotic organizations: wellfunctioning individuals in vibrant companies». Human Relations, 54(1):101111.

Kravetz, D. (1990). La revolución de los recursos humanos. Deusto.

León, J. M. (2000). «El modelo biopsicosocial o la concepción social de las acciones de salud. Una guía para la elaboración de la Psicología Social de la Salud». En A. Ovejero (ed.). La psicología social en España al filo del año 2000: balance y perspectivas. Madrid, Biblioteca Nueva.

León, J.M. y Jarana, L. (1992). «Una perspectiva psicosocial en los servicios de salud». En F. Gil, L. Jarana y J. M. León (coords.). Habilidades sociales y salud (pp. 17-29). Madrid: Eudema.

Ley 54/2003, de 12 de diciembre, de reforma del marco normativo de la prevención de riesgos laborales (BOE de 13 de diciembre de 2003).

Ley de Prevención de Riesgos Laborales. 31/1995 de 8 de Noviembre. (B.O.E. de 10 de Noviembre de 1995).

Lluch, M. T. (1999). Construcción de una escala para evaluar la salud mental positiva. Barcelona, Tesis doctoral.

Lowe, G.S.; Schellenderg, G., y Shannon H.S. (2003). "Correlates of Employees Perceptions of a Healthy Work Environment». American Journal of Health Promotion. Jlyu/August 2003, 17(6):390-399.

Lowry, C.B. y Hanges, P.J. (2008). «What is the Healthy Organization? Organizational Climate and Diversity Assessment: A Research Partnership». Libraries and the Academy, 8(1):1-5.

MacIntosh, R.; MacLean, D., y Burns, H. (2007). «Health in Organization: Towards a Process-Based View». Journal of Management Studies 44(2): 206221.

Maslow, A.H. (1971). La personalidad creadora. Kairos.

- (2005). El Management según Maslow. Barcelona: Paidós.

Mateu, M. (1984). La nueva organización del trabajo. Barcelona: Hispano Europea.

Mejías, A.; Carbonell, E. J.; Gimeno, M. A., y Fidalgo, M. (2011). Procedimiento de solución autónoma de los conflictos de violencia laboral (I). Madrid: INSHT.

Mintzberg, H. (1984). La estructuración de las organizaciones. Ariel Economía. Mintzberg, H. (1991). Diseño de organizaciones eficientes. Buenos Aires: El Ateneo.

Morgan, G. (1986). Imágenes de la organización. Ra-Ma: Madrid. 
Morin, E. (1994). Introducción al pensamiento complejo. Barcelona: Gedisa Editorial.

Murphy, L. R. y Cooper, C. L. (2000). «Models of healthy work organizations». En Murphy, L. R. y Cooper, C. L. (eds.) (2000). Healthy and productive work. An international perspective. Londres: Taylor\&Francis.

Navajas, J. (2003). De la calidad de vida laboral a la gestión de la calidad. Una aproximación psicosocial a la calidad como práctica de sujeción y dominación. Atenea Digital. En línea: <http://psicologiasocial.uab.es/athenea/index. php/atheneaDigital/article/viewArticle/108/108>.

O’Leary-Keelly, A. M.; Griffin, R. W., y Glew, D. J. (1996). «Organizationmotivated aggression: A research framework». En Academy of Management Review, 21(1):225-253.

Olabarrieta, J.C. (2010). Global Workforce Study 2010. Principales conclusiones. Towers Watson.

Organización Mundial de la Salud (OMS) (1978). Declaración de AlmaAta. Conferencia Internacional sobre Atención Primaria de Salud, Alma-Ata, URSS, 6-12 de septiembre.

- (1986). Carta de Ottawa para la promoción de la salud. Ginebra: OMS.

- (1997). Declaración de Luxemburgo sobre Promoción de la salud en el lugar de Trabajo. Luxemburgo. Documento técnico OMS. En línea: <http://www. ver.is/whp/en/luxdeclaration.html>.

- (1998). Promoción de la salud. Glosario. Ginebra: OMS.

- (2010). Entornos laborales saludables: fundamentos y modelo de la OMS. Contextualización, prácticas y literatura de apoyo. Suiza: OMS.

Organización Internacional del Trabajo (OMT), Consejo Internacional de Enfermeras, Organización Mundial de la Salud (OMS) e Internacional de Servicios Públicos (2002). Directrices marco para afrontar la violencia laboral en el sector de la salud. Ginebra: OIT.

- (2005). La violencia en el trabajo. Una carga costosa. En línea: <http://www. ilo.org/public/spanish/region/ampro/cinterfor/temas/gender/doc/cinter/ pacto/cue_gen/viol_tra.htm >. [Consultado el 12 de julio de 2006]

Ovejero, A. (2003). «Globalización y condiciones de vida laboral». Encuentros en Psicología Social. 1(5):184-186.

Paoli, P. y Merllié, D. (2001). Third European survey on working conditions 2000. Luxembourg: Office for Official Publications of the European Communities/ European Foundation for the Improvement of Living and Working Conditions. 
Parent-Thirion, A.; Fernández, E.; Hurley J*, y Vermeylen, G. (2007). Fourth European Working Conditions Survey. Dublin: European Foundation for the Improvement of Living and Working Conditions.

Parra, F. (1993). «Calidad de vida y sistema de indicadores». En Garmendia, J.A. y Parra, F. Sociología industrial y de los recursos bumanos. Madrid: Taurus Universitaria.

Patrus, R. (2007). «La empresa éticał ¿un nuevo paradigma? Condiciones, desafíos y riesgos del desarrollo de la business ethics». Revista Gestão e Planejamento, $8(1) \div 16-33$.

Peiró, J.M. y Rodríguez, I. (2008). «Estrés laboral, liderazgo y Salud organizacional». Papeles del Psicólogo. 29(1):68-82.

Píriz, R. (2009). ¿Hablamos de la responsabilidad social corporativa o de la responsabilidad corporativa con la sociedad? En línea: <http://www.microsoft.com/ business/smb/es-es/rrhh/responsabilidad-corporativa.mspx $>$.

Porter, M. (1987). From Competitive Advantage to Corporate Strategy. Harvard Business Review.

Rodríguez, A. (1992). Las organizaciones a debate. Una perspectiva psicosociológica. Universidad de Granada: Granada.

Romay, J*; García-Mira, R., y Losada, M. D. (2003). «La aportación de los programas de salud a la calidad de vida laboral en las organizaciones». Encuentros de Psicología Social, 1(5):334-338.

Salanova, M. (2008). «Organizaciones saludables y desarrollo de recursos humanos». Revista de Trabajo y Seguridad Social, (303):179-214.

Schmidt, W. C.; Welch, L., y Wilson, M. G. (2000). «Individual and organizational activities to build better health». En Murphy, L. R.y Cooper, C. L. (eds.) (2000). Healthy and productive work. An international perspective. Londres: Taylor\&Francis.

Scoтt, W.R. (2003). Organizations: rational, natural and open systems. Upper Saddle River, NJ, Prentice-Hall.

Segurado, A. y Agulló, E. (2002). «Calidad de vida laboral: hacia un enfoque integrador desde la Psicología Social». Psicothema, 14(4):828-836.

Senge, P. (1990). La quinta disciplina. Granica: Barcelona.

- (2001). «Las personas no son recursos». Entrevista concecida a la revista Executive Digest, julio, pp. 58-62.

Sparks, K.; Faragher, B*, y Cooper, C. L. (2001). «Well-being and occupational health in the 21st century workplace». Journal of Occupational and Organizational Psychology, (74):489-509. 
Stiglitz, J. E. (2002). «Empleo, justicia social y bienestar en la sociedad». Revista Internacional del Trabajo, (121): 1-2, 9-31.

Stresa Declaration on Workers Health (2006). Participants, 7th Meeting of the WHO Collaborating Centres in Occupational Health, Stresa, Italia, 8-9 Junio 2006. En línea: <http://www.who.int/occupational_health/ en/> [22 Septiembre de 2009].

Tарлсотт, D. (2011, on-line). «Top trends and developments for 2011». Enero.

Tetrick, L. E. (2002). «Individual and organizational health». Historical and Current Perspectives on Stress and Health, (2):117-141.

Traid, V;; Giner, D.; Castillo, C.; Misiego, J., y López, I. (2005). «Revisión histórica del modelo biopsicosocial». Interpsiquis. En línea: <http://www.psiquiatria.com/articulos/ depresion/20229/>.

Vandenberg, R. J.; Park, K.; Dejoy, D. M.; Willson, M. G., y GriffinBlake, C. S. (2002). "The healthy work organization model: Expanding the view of individual health and well being in the workplace». En P. L. PErrewe y D. C. Ganster (eds.), Historical and current perspectives on stress and health. Oxford: Elsevier.

Vilaseca, B. (2010). «Economía_conscienteः ¿Es rentable ser ético?». En línea: <http://pymesostenible.es/2010/02/14/economia-consciente\%C2\%BFesrentable-ser-etico/>. Feb 14, 2010. Archivado en: Noticias: Medios_Informativos y Organizaciones.

Weiler, A. (2006). Annual Review of Working Conditions in the EU: 2005-2006. Dublin: European Foundation for the Improvement of Living and Working Conditions.

Willson, M. G.; DeJoy, D. M.; Vandenberg, R. J; Richardson, H. A., y McGrath, A. L. (2004). «Work Characteristics and Employee Health and Wellbeing: Test of a Model of Healthy Work Organization». Journal of Occupational and Organizational Psychology, (77):565-588.

Zapf, D., Knorz, C. y Kulla, M. (1996). «On the Relationship between Mobbing factors, and Job Content, Social Work Environment, and Health Outcomes». European Journal of Work and Organizational Psychology, $5(2) \div 215-237$. 